\title{
Automatic Three-Dimensional Segmentation of MR Images Applied to the Rat Uterus
}

\author{
Ayelet Akselrod-Ballin ${ }^{1}$, Erez Eyal ${ }^{1,2}$, Meirav Galun ${ }^{1}$, Edna Furman-Haran ${ }^{2}$, John Moshe Gomori ${ }^{3}$, \\ Ronen Basri ${ }^{1}$, Hadassa Degani ${ }^{2}$, Achi Brandt ${ }^{1}$ \\ ${ }^{1}$ Computer Science and Applied Math, ${ }^{2}$ Biology Regulation, Weizmann Institute of Science, \\ Rehovot, ${ }^{3}$ Neuroradiology and MRI, Hadassah University Medical Center, Jerusalem, Israel
}

\begin{abstract}
We introduce an automatic 3D multiscale automatic segmentation algorithm for delineating specific organs in Magnetic Resonance images (MRI). The algorithm can process several modalities simultaneously, and handle both isotropic and anisotropic data in only linear time complexity. It produces a hierarchical decomposition of MRI scans. During this segmentation process a rich set of features describing the segments in terms of intensity, shape and location are calculated, reflecting the formation of the hierarchical decomposition. We show that this method can delineate the entire uterus of the rat abdomen in 3D MR images utilizing a combination of scanning protocols that jointly achieve high contrast between the uterus and other abdominal organs and between inner structures of the rat uterus. Both single and multi-channel automatic segmentation demonstrate high correlation to a manual segmentation. While the focus here is on the rat uterus, the general approach can be applied to recognition in $2 \mathrm{D}, 3 \mathrm{D}$ and multi-channel medical images.
\end{abstract}

Keywords: Segmentation, MRI, Small animal imaging, Uterus, Rat

\section{INTRODUCTION}

Identification of three-dimensional (3D) anatomical structures is an essential problem in medical image processing. It is a crucial step in the analysis of medical images, supporting tasks such as diagnosis, visualization, quantification, registration, reconstruction, tracking and more. The aim in image segmentation is to partition the image into its constituent parts. The desired segmentation is dictated by the type of images dealt with, their modality and anatomical structures and by the task involved. It is possible to perform manual delineation, however in cases of 3D and multi spectral data this is extremely difficult. For example, in the rat uterus model, the uterine horns are embedded in the abdomen in such a way that it is complicated to identify each plane of the crimped horns and differentiate them from the intestines and ovaries. Additionally, performing manual segmentation is time consuming and does not meet the required accuracy and reproducibility demands. Indeed, while magnetic resonance imaging (MRI) offers high spatial resolution, soft tissue contrast and multiple contrast mechanisms which can be simultaneously exploited to obtain multi-spectral 3D digital data, MRI is also susceptible to artifacts such as partial volume effect (related to the finite spatial resolution), field inhomogeneity, chemical-shift, and motion [19] posing significant difficulties on automatic segmentation.

Reviews of MRI segmentation approaches can be found in ([2], [15], [18]). Here we briefly list some of the common methods and their characteristics. Region-based and edge-based segmentation techniques emphasize respectively within-region similarities and between-region differences using appropriate features. Classical region based strategies include region growing, region splitting, and split and merge algorithms. Low level region based and edge based techniques, which exploit only local information for each voxel and do not incorporate global shape and boundary constraints are limited when dealing with automatic MRI segmentation. Classification techniques usually assign class labels to voxels in the volume according to feature values. Common classification techniques include k-means, fuzzy cmeans, k-nearest neighbors (K-NN), clustering algorithms ([2],[7]), decision trees [4], and artificial neural network (ANN)[24]. Methods such as deformable models are parameterized curves or surfaces which evolve towards the desired location through an energy minimization process. The energy function is based on external forces derived from the data (high gradient for instance) and internal forces related to the geometry of the contour itself (typically the curvature). This model was introduced by Kass et al. [12], and recent surveys of these methods in medical image analysis can be found in ([13],[14]). These techniques benefit from consideration of global prior knowledge about expected shape. Yet their limitations come from their dependence on initialization and from the computation time required for 3D, multichannel segmentation. Statistical models have also been widely used for segmentation of MRI. Wells et al [22], 
pioneered the expectation maximization (EM) segmentation of MRI images. The approach modeled the intensity of brain tissues by a Gaussian Mixture Model (GMM) and iteratively estimated the intensity inhomogeneity correction (bias field) and classified intensity regions in the brain. This approach was extended by other studies to include spatial considerations utilizing a brain atlas ([15], [20], [21]).

This paper describes a general framework for automated segmentation of MRI. Our fast, 3D segmentation algorithm produces a full hierarchy of segments, expressed by an irregular pyramid, in only a linear time complexity. Unlike other approaches which are based mainly on intensity as a feature, our approach includes a thorough multiscale set of measurements, computed and used throughout the segmentation process, and characterizing the resulting segments. The enlarged feature set supports enhanced analysis of the detected structures and allows incorporation of spatial, neighborhood and geometric considerations into the segmentation process, enabling detection of various anatomical structures at different scales. We applied the approach to the analysis of in vivo MR images of the rat uterus. The rat uterus is a commonly used model for hormonal regulation research. However, due to its $3 \mathrm{D}$ complex structure manual uterus segmentation is highly time consuming. Consequently, only a limited number of in vivo uterine MRI studies in experimental animals have been conducted. Current uterine experiments usually involve sacrificing the animals and extracting uterine tissues ([10], [23]). Developing non-invasive imaging techniques to monitor the uterus in vivo can allow spatial and temporal follow up on the same animal model and enhance precision as well as reproducibility. While numerous segmentation approaches have been applied to the analysis of the human brain, we are not familiar with any automatic segmentation algorithm applied to the detection of the rat uterus in MRI. In the present paper we focus on a detailed description of the segmentation methods. A future work will describe in more detail their use to analyze time courses of functional and molecular imaging of uterine tissue.

\section{METHODS}

This study extends the Segmentation by Weighted Aggregation (SWA) algorithm ([8], [16], [17]), developed for 2D natural images, in order to perform segmentation of an aligned set of MR images by a multiscale 3D multi-channel approach (also referred to as multi-modal, multi-sequence, or multi-spectral). In this section we review the original SWA algorithm along with our modifications. Given a 3D MRI, we construct a graph in which every voxel is a node and neighboring voxels are connected by an edge. A weight is associated with each edge reflecting the similarity between the corresponding voxels. Subsets of the nodes in the graph which approximately minimize a normalized-cutlike measure may indicate the segments in the image. To find the minimal cuts in the graph, the graph can be coarsened through a recursive process of weighted aggregation derived from algebraic multi-grid (AMG)[3] coarsening procedures. This coarsening process induces a pyramid structure over the image. At each coarse scale there are about half as many nodes as in the next finer scale. These coarse nodes, which represent aggregates of voxels, do not necessarily lie on a grid. Segments that are distinct from their surroundings emerge as salient nodes (weakly connected to others) in their corresponding scale. The fuzziness (weighted relation) relations of the aggregation allow the algorithm to avoid wrong local decisions and to detect segments based on a global saliency measure.

\section{Graph Terminology}

Given a 3D MRI data set, a 6-connected graph $G=(V, W)$ is constructed from the 3D image as follows. Each voxel $i$ is represented by a graph node $i$, so $V=\{1,2, \ldots, N\}$ where $N$ is the number of voxels. A weight $\omega_{i j}$ is associated with each pair of neighboring voxels. The 6-connected graph adds for every node $i$ two additional neighbors from the neighboring slices, unlike the 2D approach which analyzes each slice separately by a 4-neighbor graph. The weight $\omega_{i j}$ reflects the contrast between the two neighboring voxels $i$ and $j$. For example in the single channel case it is formulated as:

$$
\text { (1) } \quad \omega_{i j}=e^{-\alpha\left|I_{i}-I_{j}\right|}
$$

where $I_{i}$ and $I_{j}$ denote the intensities of the two neighboring voxels, and $\alpha$ is a positive constant ( $\alpha=10$ in our experiments).

Our way to evaluate segments is based on a normalized-cut-like measure, which is defined as follows. Every segment $S \subseteq V$ is associated with a state vector $u=\left(u_{1}, u_{2}, \ldots, u_{N}\right)$, representing the assignments of voxels to a segment $S$ : 


$$
u_{i}=\left\{\begin{array}{lc}
1 & i \in S \\
0 & i \notin S
\end{array}\right.
$$

The saliency associated with $S$ is defined by the normalized-cut-like cost

$$
\Gamma(S)=\frac{\sum_{i>j} \omega_{i j}\left(u_{i}-u_{j}\right)^{2}}{\sum_{i>j} \omega_{i j} u_{i} u_{j}}
$$

which sums the weights along the boundaries of $S$ divided by its internal weights. Segments that yield small values of $\Gamma(S)$ are considered salient. In matrix notation $\Gamma$ can be written as

$$
\Gamma(S)=\frac{u^{T} L u}{\frac{1}{2} u^{T} W u}
$$

where $\mathrm{L}$ is the Laplacian matrix derived from $\mathrm{W}$. Our objective is to find segments $\mathrm{S}$ characterized by a small value of $\Gamma(S)$. For that end we construct a coarse version of the graph. This coarse version is constructed so that we can use salient segments in the coarse graph to predict salient segments in the fine graph using only local calculations. This coarsening process is repeated recursively, constructing a full pyramid structure. Each node at every scale represents an aggregate of voxels. Each segment $\mathrm{S}$, which is a salient aggregate (i.e., $\Gamma(S)$ is low) emerges as a single node at a certain coarsening step.

The coarsening procedure proceeds recursively as follows. Starting from the given graph $G^{[0]} \equiv G$, we recursively coarsen the cut minimization problem, creating a sequence of decreasing size graphs $G^{[0]}, \ldots, G^{[k]}$. At each scale we seek for nodes with low $\Gamma$, representing salient aggregates. As in the general AMG setting, the construction of a coarse graph from a given one is divided into three stages: first a subset of the fine nodes is chosen to serve as the seeds of the aggregates (the latter being the nodes of the coarse graph), then the rules for interpolation are determined, thereby establishing the fraction of each non-seed node belonging to each aggregate, and finally the weights of the edges between the coarse nodes is calculated.

Coarse seeds: The construction of the set of seeds $\mathrm{C}$, and its complement denoted by F, is guided by the principle that each F-node should be "strongly coupled" to $\mathrm{C}$. To achieve this objective we start with an empty set $\mathrm{C}$, hence $\mathrm{F}=\mathrm{V}$, and sequentially transfer nodes from $\mathrm{F}$ to $\mathrm{C}$ until all the remaining $i \in F$ satisfy $\sum_{j \in C} \omega_{i j} \geq \beta \sum_{j \in V} \omega_{i j}$, where $\beta$ is a parameter (in our experiments $\beta=0.2$ ).

Interpolation: we define for each node $i \in F$ a coarse neighborhood $N_{i}=\left\{j \in C, \omega_{i j}>0.001\right\}$. Let $\mathrm{I}(\mathrm{j})$ be the index in the coarse graph of the node that represents the aggregate around the seed whose index at the fine scale is $j$. The classical AMG interpolation matrix $P$ of size $N x n$ where $n=\mid C l$ is defined by

$$
P_{i I(j)}= \begin{cases}\frac{\omega_{i j}}{\sum_{k \in N_{i}} \omega_{i k}} & \text { for } i \in F, j \in N_{i} \\ 1 & \text { for } i \in C, j=i \\ 0 & \text { otherwise }\end{cases}
$$

It satisfies $u \approx P U$ where $U=\left(U_{1}, U_{2}, \ldots, U_{n}\right)$ is the coarse level state vector. $P_{i l}$ represents the likelihood of $i$ to belong to the $I$-th aggregate. 
The coarse problem: Following the weighted aggregation scheme [3], the edge connecting two coarse aggregates $p$ and $\mathrm{q}$ is assigned with the weight:

$$
\omega_{p q}^{\text {coarse }}=\sum_{k \neq l} P_{k p} \omega_{k l} P_{l q}
$$

Namely the coupling weight between a pair of coarse aggregates is the weighted sum of the coupling weights between their subaggregates. Attaching the scale superscript $G^{[s]}=\left(V^{[s]}, W^{[s]}\right)$. Equation (6) means $W^{[s]}=P^{[s] T} W^{[s-1]} P^{[s-1]}$, where $P^{[s-1]}$ is the interpolation matrix $P$ from $V^{[s]}$ to $V^{[s-1]}$. Note that since $u^{[s-1]} \approx P U^{[s-1]}$ the relation (4) inductively implies that similar expression approximates $\Gamma$ at all scales.

During the weighted aggregation scheme the algorithm computes 3D internal statistics of aggregates, called aggregative properties, which will be used to evaluate similarities between neighboring aggregates and which will also be available for further analysis at the end of the segmentation process. Namely, for each aggregate $\mathrm{i}$ emerging at a certain scale s, we calculate a set of aggregative properties. An aggregative property can be expressed as a weighted average over the aggregate $i$ of a property that has first appeared at a scale $r$, where $r \leq s$. The scale $s$ is termed the aggregate scale and the scale $r$ is called the property scale. The interpolation matrices (Eq. (5)) are used recursively to accumulate quantities for the aggregates properties. At each scale $s$ the similarity matrix $W^{[s]}$, inherited from finer aggregate scales (Eq. (6)), is modified to account for the similarities arising from the set of aggregative properties obtained from multiple property scales. Specifically, in the present work the segmentation process incorporated aggregative properties that describe average intensity, variance of average intensity and shape properties specified by applying principal component analysis to the covariance matrix of the aggregate. However, additional statistical properties are computed throughout the segmentation for all the aggregates and are therefore available for the uterus segment analysis.

The performance of the algorithm is $\mathrm{O}(\mathrm{N})$, linear in the number of voxels. On each scale of the pyramid $O\left(N^{[s]}\right) \approx O\left(N / 2^{s}\right)$ operations are performed, so that the complexity of one bottom up process is $\mathrm{O}(\mathrm{N})$.

\section{3D segmentation of anisotropic data}

MRI data is usually anisotropic, less vertically resolved (e.g. $0.5_{\mathrm{mm}}$ thickness compared to $0.27_{\mathrm{mm}}$ by $0.27_{\mathrm{mm}}$ in-plane resolution). Therefore, starting with $G^{[0]}$ the coarsening process is initially performed only in $2 \mathrm{D}$ (e.g. in each horizontal plane) rather than 3D. Except that the weighted aggregation scheme is applied to all the couplings. Consequently the between-plane couplings are only updated by Eq. 6, but have no other effect on the initial segmentation. Thus, nodes from different slices are initially (in the first coarsening stages) not aggregated together although they may be "strongly coupled". This is done until a scale is reached where the horizontal distances between nodes are comparable to the vertical ones, upon where the full 3D process starts.

For instance, in our experiments the $G^{[0]}$ grid refers to data with $0.5_{\mathrm{mm}}$ slice thickness versus $0.27_{\mathrm{mm}} \mathrm{x} 0.27_{\mathrm{mm}}$ in-slice resolution; Every coarsening step of the SWA algorithm typically reduces the number of nodes by a factor of 2.5-3. Consequently, if we apply the algorithm to a $2 \mathrm{D}$ slice, the distance between neighboring nodes in a slice grows at every level by a $\sqrt{2} .5-\sqrt{3}$ factor on average, so two coarsening steps are needed to bring the inner- and inter-slice distances to be roughly equal.

Comparing our former purely 2D segmentation with our current 3D segmentation reveals that the information from neighboring slices is vital for accurate segmentation, especially in the case of the rat uterus horns that consist of elongated structures which are only partially included in every slice. The $3 \mathrm{D}$ approach significantly improves the detection of the entire uterus, since the real appearance of structures is three dimensional.

\section{Multi-Channel segmentation}

One of the unique characteristics of MRI is the multiple methods to produce contrast between different tissues or organs in the image. To incorporate several channels of data, the graph structure is modified so that every entry (a voxel intensity, or an aggregative property) is replaced by a vector of entries (each corresponding to a different channel). The initialization step is modified to consider the intensity contrast in all channels, and the different sets of aggregative 
features for every channel are all used to modify the couplings at coarser levels. Our preliminary results show that the combination of several channels in the segmentation process can lead to superior results which usually can not be achieved by considering just one channel.

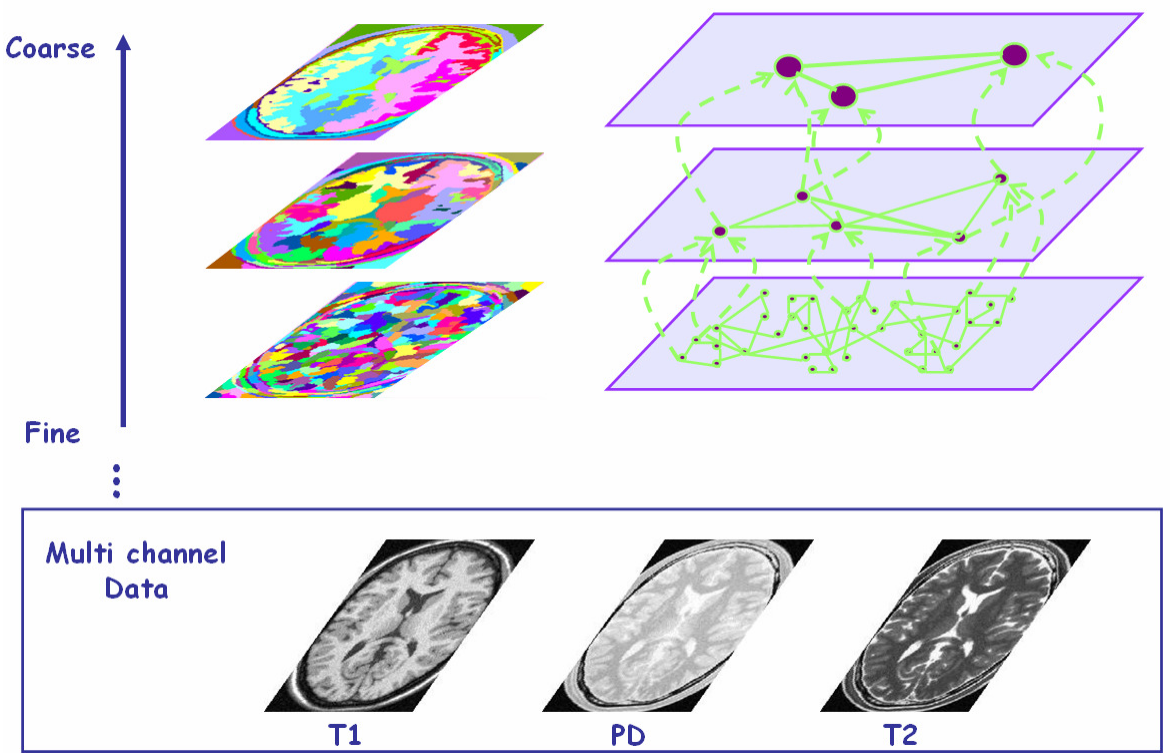

Figure 1: Illustration of the segmentation pyramid hierarchy. The pyramid is constructed from fine scale to coarse scale based on the multi-channel 3D input data (e.g, T1, T2, PD channels).
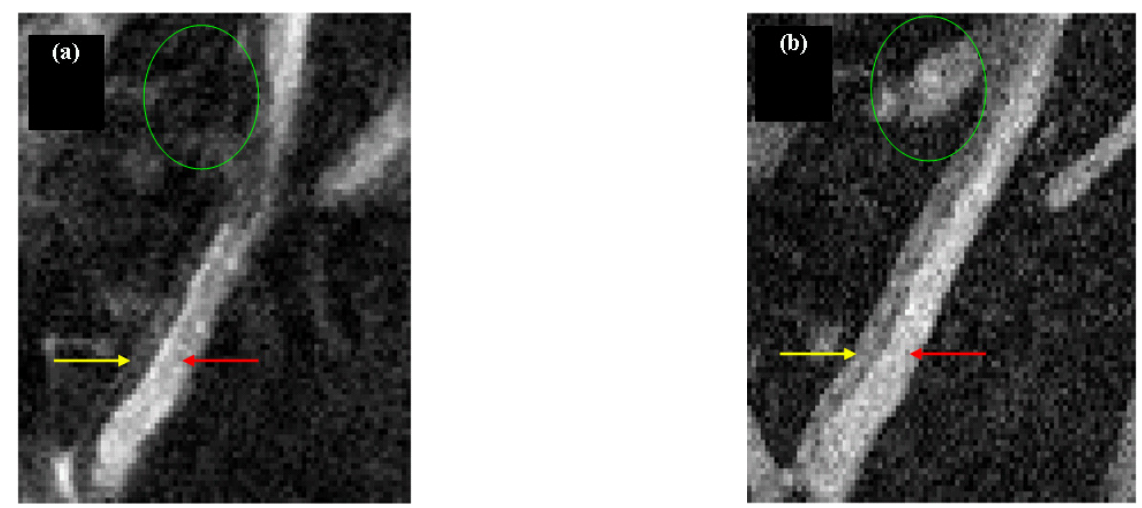

Figure 2: An example of a central coronal slice of the abdominal cavity: The right uterus horn imaged by (a) T2w and (b) PDw protocols. The red and yellow arrows point to the endometrial and the myometrial tissue respectively on both channels. Part of the intestine organ is circled (green).

\section{EXPERIMENTS}

\section{MR image acquisition:}

The MR experiments were performed in female, ovariectomized, LEW/NHSD rats (6-8 month). Water imbibition [5] was artificially induced by subcutaneous (s.c.) injection in the periscapular region of $17-\beta$ estradiol $(50 \mu \mathrm{gr} / \mathrm{kg} \mathrm{BW})$ dissolved in sesame oil, 24 hours before the experiments. In all the MRI experiments, rats were anesthetized by 
inhalation of 1.5\% Isoflurane in an $\mathrm{O} 2 \mathrm{~N} 2 \mathrm{O}$ (3:7) mixture, applied through a nose. All animal procedures were approved by the Weizmann Institute's Animal Care and Use Committee.

MR images were acquired with a $4.7 \mathrm{~T} / 30 \mathrm{~cm}$ bore Biospec spectrometer (Bruker, Germany), equipped with a ${ }^{1} \mathrm{H}$ radio frequency coil with an inner diameter of $7.5 \mathrm{~cm}$. Coronal MR images tilted in 10 approximately covering the whole abdominal cavity. The spatial resolution for all sequences was $0.27 \times 0.27 \times 0.5 \mathrm{~mm}^{3}$, using a matrix of $256 \times 256 \times 15$. Fat suppression was applied in all protocols by applying a 90 degree Gauss shaped preparation pulse $700 \mathrm{~Hz}$ off resonance (SW of $600 \mathrm{~Hz}$ ) followed by a dephasing gradient. The protocols used as channels for the automatic segmentation were:

1. T2 weighted (T2w) 2D Rapid Acquisitions with Refocused echoes (RARE) [11] with TE/TR of 47.2/3200 ms, a rare factor of 4 and an acquisition time of $3 \mathrm{~min}$.

2. Proton density weighted (PDw), 2D fast low-angle shot gradient echo with TE/TR of $4.2 / 500 \mathrm{~ms}, 30^{\circ}$ flip angle, and an acquisition time of 3 min.

\section{Single and multi channel segmentation experiment:}

Single channel 3D segmentation was performed on the T2w and PDw channels separately and multi-channel segmentation was performed by combining both channels. Figure 2 presents the right uterus horn imaged by T2w and PDw protocols. The figure demonstrates the different characteristics of the two protocols in detecting the inner structure of the rat uterus. The image shows that the rat uterus horn is composed of two anatomical layers, the myometrium and the endometrium, which correspond to the outer and inner layers of the horn respectively. The T2w sequence distinctly enabled delineating the endometrium (Figure 2.a) and the PDw sequence allowed capturing the entire uterus, endometrium and myometrium. However, the latter sequence results in low contrast between the uterus and the intestine (Figure 2.b). Based on these MR protocols properties we designed an experiment that enabled us to manually and automatically perform uterus segmentation.

\section{RESULTS}

To evaluate the algorithm performance, manual segmentation was performed for both channels separately. Quantitative comparison based on common measures ([6],[9],[24]) for spatial overlap are presented in Table 1,2.

\begin{tabular}{|l|l|c|}
\hline & $|S \cap R| /|R|$ & $2 \cdot|S \cap R| /(|R|+|S|)$ \\
\hline $\begin{array}{l}\text { Endometrial segmentation based on single- } \\
\text { channel T2w }\end{array}$ & $0.91 \pm 0.04$ & $0.88 \pm 0.01$ \\
\hline
\end{tabular}

Table 1: Validation results for segmentation of the endometrium tissue in the rat uterus. Automatic segmentation (S) is compared with ground truth manual reference $(\mathrm{R})$, averaged over 4 uteral structures.

\begin{tabular}{|l|l|c|}
\hline & $|S \cap R| /|R|$ & $2 \cdot|S \cap R| /(|R|+|S|)$ \\
\hline $\begin{array}{l}\text { Myometrial and Endometrial segmentation } \\
\text { based on single-channel PDw }\end{array}$ & $0.95 \pm 0.01$ & $0.57 \pm 0.14$ \\
\hline $\begin{array}{l}\text { Myometrial and Endometrial segmentation } \\
\text { based on Multi-channel }\end{array}$ & $0.91 \pm 0.03$ & $0.76 \pm 0.02$ \\
\hline
\end{tabular}

Table 2: Validation results for single and multi channel segmentation of the entire rat uterus. Automatic segmentation $(\mathrm{S})$ is compared with ground truth manual reference $(\mathrm{R})$, averaged over 4 uteral structures.

Single Channel Segmentation: As expected, due to the water imbibition effect the segmentation of the T2w data achieved accurate results for the endometrial tissue while the myometrial tissue could not be differentiated (Table 1, Figure 3). The segmentation based on the PDw data alone achieved poor results due to low contrast between the uterus and intestine (Table 2, Figure 4 A-C). Segmentation execution, with our non optimal implementation, for 200x200x15 voxels took $40 \mathrm{sec}$ on a standard $\mathrm{P} 43 \mathrm{GHz} \mathrm{PC}$. 

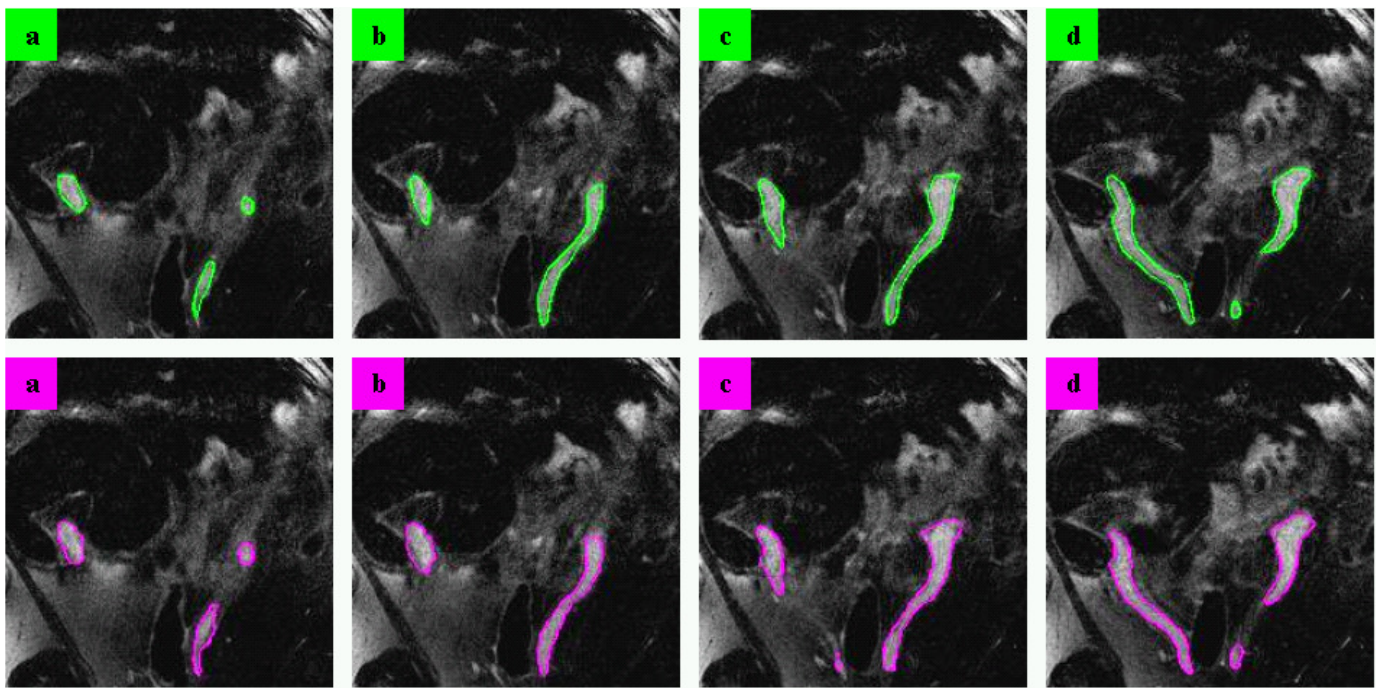

Figure 3: Comparison of manual (green) and automatic (magenta) segmentation results for the endometrium tissue. The results presented on a set of 2D slices (A-D ventral to dorsal), were obtained with a single channel T2w approach.
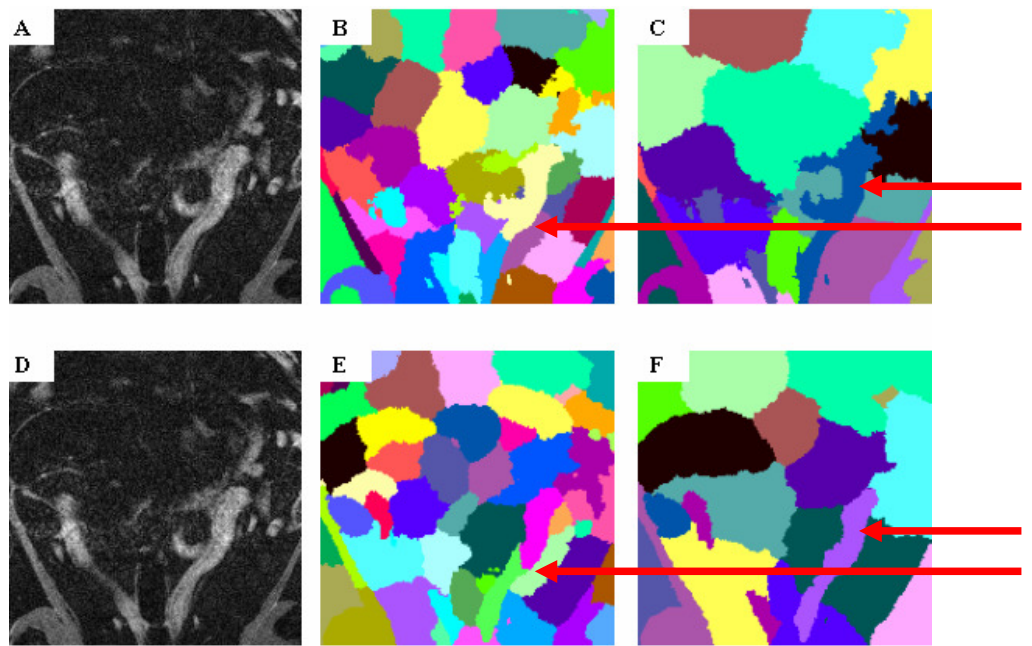

Figure 4: Segmentation by single and multi-channel approaches for the entire uterus displayed on a PDw coronal slice (A,D). Singlechannel results on PDw show how part of the intestine is added to the ROI at levels 7,8 (B,C). While for the multi-channel approach the two subpart of the right horn are formed at scale 6(E) and at scale 7(F) they connect to successfully detect the entire uterus.

(a)

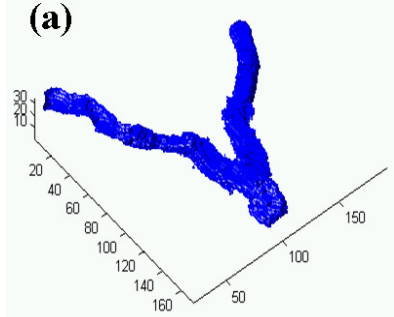

(b)

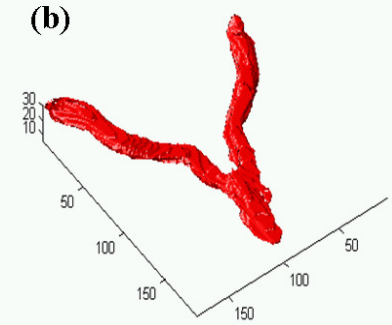

Figure 5: 3D view of multi channel segmentation results of the entire uterus: (a) automatic and (b) manual. 
Multi-Channel Segmentation: The multi-channel segmented ROI calculated using the combined T2w and PDw images yielded an ROI covering most of the uterus volume regardless of the inner structure of the horns (Figure 5). The T2 weighted image served as a backbone for the segmentation, while the data derived from the PD image referred to the peripheral details (Figure 4, D-F). The execution time for segmentation of both channels was 60 sec.

Consequently, applying the multi-channel approach to these channels increased the detection of the entire uterus compared to the single channel segmentation. The validation results presented in Table 1,2 show the high accuracy rates obtained by the automatic segmentation in detecting either the entire uterus by the multi-channel approach or just the endometrium layer by the single $\mathrm{T} 2 \mathrm{w}$ channel approach. The similarity measure (second column in Table 1,2) corresponds to kappa statistics where a value $\geq 0.7$ is considered as a good agreement [24].

\section{DISCUSSION}

We present a novel 3D automatic segmentation algorithm applied for the accurate detection of the uterus structure. The segmentation algorithm is an unsupervised, multiscale algorithm that can be applied to anisotropic data and enables integration of unlimited MR pulse sequences with no constraint on the scan protocol. Quantitative validation results show the high accuracy of the automatic segmentation compared with the manual delineation, in both the mutli-channel segmentation of the entire uterus, and the single channel $\mathrm{T} 2 \mathrm{w}$ segmentation of the only endometrium layer.

A number of additional modifications based on high level knowledge of the uteral structure could improve the overall performance of the approach. For example, the algorithm can easily incorporate knowledge of the expected uterus shape, using the already computed aggregative properties (e.g. length, width, orientation) to modify the similarities between neighboring aggregates. This can assist the elongated subparts of the uterus horns with well defined orientation to merge together and to improve detection rate. Also some post processing morphology rules based on the prior knowledge of anatomy and the different channels properties can be employed at the end of the process to increase precision. Future work will test the usefulness of such modifications.

Finally, the presented approach, originally developed for segmentation of MR human brain images [1], can be generalized to other structures and tasks. The development of non-invasive imaging techniques to monitor anatomical structures may allow temporal follow up on the same animal and enhance accuracy, reproducibility and efficiency. We show that combining the 3D information from different MRI pulse sequences is very useful for improving the accuracy of the segmentation. The approach produces a full hierarchy of segments in time that is linear in the dataset size and shows low sensitivity to some MRI artifacts, making it practical for various medical image segmentation experiments. Such an algorithm may also be useful for segmentation of MR angiography and virtual colonoscopy.

\section{REFERENCES}

[1] A. Akselrod-Ballin, M. Galun, M. J. Gomori, M. Filippi, P. Valsasina, R. Basri, and A. Brandt, "Integrated Segmentation and Classification Approach Applied to Multiple Sclerosis Analysis", submitted.

[2] J.C. Bezdek, L.O. Hall, and L. P. Clarke, "Review of MR image segmentation techniques using pattern recognition”, Medical Physics, 20, 1033-1048, 1993.

[3] A. Brandt, S. McCormick and J. Ruge, "Algebraic multigrid (AMG) for automatic multigrid solution with application to geodetic computations", Inst. for Computational Studies, POB 1852, Fort Colins, Colorado, 1982.

[4] L. Breiman and J.H. Olshen and C.J. Stone, Classification And Regression Trees, Wadsworth Int. Group, 1984.

[5] P. Carthew, R.E. Edwards, B.M. Nolan, M.J. Tucker, L.L. Smith. "Compartmentalized uterotrophic effects of tamoxifen, toremifene, and estradiol in the ovariectomized Wistar (Han) rat.” Toxicol Sci., 48(2), 197-205, 1999.

[6] L.R. Dice, "Measures of the amount of ecologic associating between species", Ecology, 26(3), 297-302, 1945.

[7] R.O. Duda and P.E. Hart, Pattern classification and scene analysis. John Wiley and Sons, 1973.

[8] M. Galun, E. Sharon, R. Basri, and A. Brandt, "Texture Segmentation by Multiscale Aggregation of Filter Responses and Shape Elements", ICCV, I:716--723, 2003.

[9] G. Gerig and M. Jomier and M. Chakos, "VALMET: A new validation tool for assessing and improving 3D object segmentation", MICCAI, 516-52, 2001. 
[10] G.S. Hamilton, T.G. Kennedy, C.J. Norley, S.J. Karlik, "Gadolinium-DTPA enhanced MRI demonstrates uterine vascular changes associated with artificially induced decidualization and ovoimplantation in rats". Magn Reson Med, 29(6), 817-2, 1993.

[11] J. Henning, A. Nauerth, H. Friedburg. "RARE imaging: a fast imaging method for clinical MR" Magn Reson Med., 3(6), 823-33, 1986.

[12] M. Kass, A. Witkin, D. Terzopoulos, "Snakes: Active contour models", International Journal of Computer Vision, 1(4), 321-331, 1987.

[13] T. McInerney and D. Terzopoulos. "Deformable Models in medical image analysis: A survey". Medical Image Analysis, 1(2), 91-108, 1996.

[14] J. Montagnat, H. Delingetter, and N. Ayache, "A review of deformable surfaces: topology, geometry and deformation, Image and Vision Computing., 19(14),1023-1040, 2001

[15] D.L. Pham, C. Xu, J.L. Prince, "Current Methods in Medical Image Segmentation", Annual Review of Biomedical Engineering, 2, 315-337, Annual Reviews, Palo Alto, CA, 2000.

[16] E. Sharon, A. Brandt, R. Basri, "Fast Multiscale Image Segmentation”, IEEE CVPR, I:70-77, 2000.

[17] E. Sharon, A. Brandt, R. Basri, "Segmentation and Boundary Detection Using Multiscale Intensity Measurements", IEEE CVPR I:469-476, 2001

[18] M. Sonka and J. M. Fitzpatrick, Handbook of Medical Imaging, Volume 2: Medical image processing and analysis, SPIE Press, Bellingham, WA, 2000.

[19] D.D. Stark, W.G. Bradley, Magnetic resonance imaging, $3^{\text {rd }}$ edition, Eds. St Louis, Mo: Mosby, 1999.

[20] K. Van Leemput, F. Maes, D. Vandermeulen, A. Colcher, P. Suetens, "Automated segmentation of ms lesions by model outlier detection", IEEE Trans. MI, 20, 677-688, 2001.

[21] X. Wei, S. K. Warfield, K. H. Zou, Y. Wu, X. Li, A. Guimond, JP 3rd Mugler, R. R Benson, L. Wolfson, H.L. .Weiner, C.R. Guttmann, "Quantitative analysis of MRI signal abnormalities of brain white matter with high reproducibility and accuracy”, J Magn Reson Imaging., 15, 203-9, 2002.

[22] W.M. Wells, W.E.L. Grimson, R. Kikinis, F.A. Jolesz. "Adaptive segmentation of MRI data”, IEEE Trans MI, 15, 429-442, 1995.

[23] Y. Yang, S. Xu, M.J. Dawson, "Measurement of water diffusion in hormone-treated rat uteri by diffusionweighted magnetic resonance imaging", Magn Reson Med, 33(5), 732-5, 1995.

[24] A. Zijdenbos, B.M. Dawant and R.A. Margolin and A.C Palmer, "Morphometric Analysis of White Matter Lesions in $\{\mathrm{MRI}\}$ : Method and Validation”, IEEE Trans. MI, 14(3), 442-453, 1994. 